\title{
CHARACTERIZATIONS OF ELEMENTS IN PRIME RADICALS OF SKEW POLYNOMIAL RINGS AND SKEW LAURENT POLYNOMIAL RINGS
}

\author{
Jeoung Soo Cheon, Eun Jeong Kim, Chang Ik Lee, and Yun Ho Shin
}

\begin{abstract}
We show that the $\theta$-prime radical of a ring $R$ is the set of all strongly $\theta$-nilpotent elements in $R$, where $\theta$ is an automorphism of $R$. We observe some conditions under which the $\theta$-prime radical of $R$ coincides with the prime radical of $R$. Moreover we characterize elements in prime radicals of skew Laurent polynomial rings, studying $\left(\theta, \theta^{-1}\right)$ (semi)primeness of ideals of $R$.
\end{abstract}

\section{Introduction}

Throughout $R$ denotes a ring with identity and $\theta: R \rightarrow R$ is an automorphism of $R$. We use $\mathbb{Z}$ to denote the ring of integers. An ideal $I$ of $R$ is called a $\theta$-ideal if $\theta(I) \subseteq I$, and is called $\theta$-invariant if $\theta(I)=I$. There are some examples of $\theta$-ideals which are not $\theta$-invariant.

Example 1.1. Let $K$ be any ring and $T=K\left[x_{i} \mid i \in \mathbb{Z}\right]$ be the free algebra over $K$ in the commuting indeterminates $x_{i}, i \in \mathbb{Z}$. Define a $K$-homomorphism $\theta: T \rightarrow T$ by $\theta\left(x_{i}\right)=x_{i+1}, i \in \mathbb{Z}$.

(1) Put $I_{1}=\sum_{i \leq-1} T x_{i}^{2} \oplus \sum_{i \geq 0} T x_{i}$. Then it is a $\theta$-ideal of $T$. However, it is not $\theta$-invariant, since $x_{0} \in I_{1} \backslash \theta\left(I_{1}\right)$.

(2) Consider the ideal $N$ of $T$ generated by the monomials $x_{i_{1}} \cdots x_{i_{n}}$, where $n \geq 2$, then it is a $\theta$-invariant ideal of $T$. Thus, $\theta$ induces an automorphism of $R=T / N \cong K \oplus \sum_{i \in \mathbb{Z}} K \bar{x}_{i}$, where $\bar{x}_{i}=x_{i}+N$. Put $I_{2}=\sum_{i \geq 1} K \bar{x}_{i}$. Then it is a $\theta$-ideal of $R$. However, it is not $\theta$-invariant since $\theta(I)=\sum_{i \geq 2} K \bar{x}_{i}$.

According to Pearson and Stephenson [4], a proper $\theta$-ideal $I$ of $R$ is called $\theta$-prime provided that if $A B \subseteq I$ for an ideal $A$ and a $\theta$-ideal $B$ in $R$, then $A \subseteq I$ or $B \subseteq I$; a proper $\theta$-ideal $I$ of $R$ is called $\theta$-semiprime provided that whenever $A$ is an ideal of $R$ and $m$ is an integer such that $A \theta^{k}(A) \subseteq I$ for all

\footnotetext{
Received June 2, 2009.

2010 Mathematics Subject Classification. 16N40, 16N60, 16 S36.

Key words and phrases. $\theta$-ideal, $\theta$-prime ideal, $\theta$-semiprime ideal, strongly $\theta$-nilpotent element, $\theta$-prime radical, prime radical, skew polynomial ring, skew Laurent polynomial
} ring. 
$k \geq m$ we have $A \subseteq I$. It is not difficult to check that a $\theta$-invariant ideal $I$ of $R$ is $\theta$-prime if and only if $A \subseteq I$ or $B \subseteq I$ whenever $A, B$ are ideals in $R$ and $m$ is an integer such that $A \theta^{\bar{k}}(B) \subseteq I$ for all integers $k \geq m$. Thus $\theta$-prime ideals are $\theta$-semiprime.

A ring is called $\theta$-prime ( $\theta$-semiprime) if the zero ideal is $\theta$-prime $(\theta$-semiprime). The (left) skew polynomial ring by $\theta$ over $R$ is denoted by $R[x ; \theta]$. Note that $\theta$ extends to an automorphism $\theta^{*}: R[x ; \theta] \rightarrow R[x ; \theta]$ defined by $\theta^{*}\left(\sum_{i \geq 0} a_{i} x^{i}\right)=\sum_{i \geq 0} \theta\left(a_{i}\right) x^{i}$ for all $\sum_{i \geq 0} a_{i} x^{i} \in R[x ; \theta]$.

We first recall the following result proved by Pearson and Stephenson.

Lemma 1.2 ([4, Proposition 1.1]). (1) $R[x ; \theta]$ is a prime ring if and only if $R$ is $\theta$-prime.

(2) $R[x ; \theta]$ is a semiprime ring if and only if $R$ is $\theta$-semiprime.

$P(R)$ denotes the prime radical of $R$ (i.e., the intersection of all prime ideals in $R$ ). Analogously we define the $\theta$-prime radical of $R$ by

$$
\bigcap\{P \mid P \text { is a } \theta \text {-invariant prime ideal of } R\},
$$

which is written by $P_{\theta}(R)$.

The prime radical of $R[x ; \theta]$ had been completely described as follows.

Lemma 1.3 ([4, Theorem 1.3]). The prime radical of $R[x ; \theta]$ is

$$
\begin{aligned}
P(R[x ; \theta]) & =\left\{\sum_{i \geq 0} a_{i} x^{i} \mid a_{0} \in P(R) \cap P_{\theta}(R) \text { and } a_{i} \in P_{\theta}(R) \text { for } i \geq 1\right\} \\
& =\left(P(R) \cap P_{\theta}(R)\right)+\sum_{i \geq 1} P_{\theta}(R) x^{i} .
\end{aligned}
$$

Corollary 1.4. $R[x ; \theta]$ is semiprime if and only if $R$ is $\theta$-semiprime if and only if $P_{\theta}(R)=0$.

Remark 1.5. For a proper $\theta$-invariant ideal $I$ of $R$, the map $\bar{\theta}: R / I \rightarrow R / I$, defined by $\bar{\theta}(a+I)=\theta(a)+I$ for $a \in R$, is an automorphism. Moreover for an ideal $P$ of $R$ with $I \subseteq P, P$ is $\theta$-prime ( $\theta$-semiprime) if and only if $P / I$ is $\bar{\theta}$-prime $(\bar{\theta}$-semiprime $)$. Thus we have $P_{\bar{\theta}}(R / I)=Q / I$ where $Q=\bigcap\{P \mid$ $P$ is a $\theta$-invariant prime ideal of $R$ and $I \subseteq P\}$.

The following lemma is an immediate consequence of Remark 1.4.

Lemma 1.6. A proper $\theta$-invariant ideal $I$ of $R$ is $\theta$-prime ( $\theta$-semiprime) if and only if $R / I$ is $\bar{\theta}$-prime ( $\bar{\theta}$-semiprime).

Proposition 1.7. Let $I$ be a proper $\theta$-invariant ideal of $R$ and $\bar{\theta}$ be the automorphism of $R / I$ defined as above. Then the following conditions are equivalent:

(1) $I$ is a $\theta$-semiprime ideal of $R$;

(2) $R / I$ is a $\bar{\theta}$-semiprime ring;

(3) $P_{\bar{\theta}}(R / I)=0$; 
(4) $I$ is the intersection of some $\theta$-invariant prime ideals of $R$;

(5) $(R / I)[x ; \bar{\theta}]$ is a semiprime ring;

(6) $I[x ; \theta]=\left\{\sum_{i \geq 0} a_{i} x^{i} \mid a_{i} \in I\right\}$ is a semiprime ideal of $R[x ; \theta]$.

Proof. (1) $\Leftrightarrow(2)$ is obtained by Lemma 1.5. Corollary 1.3 gives $(2) \Leftrightarrow(3) \Leftrightarrow(5)$. $(3) \Leftrightarrow(4)$ is proved by the argument in Remark 1.4. $(5) \Leftrightarrow(6)$ follows from the fact that $\frac{R}{I}[x ; \bar{\theta}] \cong \frac{R[x ; \theta]}{I[x ; \theta]}$.

Recall that $P(R)$ is the smallest semiprime ideal of $R$. The following is a similar result for $P_{\theta}(R)$, obtained from Proposition 1.6.

Corollary 1.8. $P_{\theta}(R)$ is the smallest $\theta$-invariant semiprime ideal, i.e., $P_{\theta}(R)$ is contained in every $\theta$-invariant semiprime ideal of $R$.

Note that $P(R)$ is the set of all strongly nilpotent elements in $R$ [2, Proposition 3.2.1]. Similarly we can characterize elements in $P_{\theta}(R)$ as follows.

An element $a$ in $R$ shall be called strongly $\theta$-nilpotent provided that for any sequence $\left(t_{n}\right)_{n=0}^{\infty}$ of positive integers such that $t_{n+1} \geq 1+\sum_{i=0}^{n} t_{i}$, and for any sequence $\left(a_{n}\right)_{n=0}^{\infty}$ in $R$ such that $a_{0}=a$ and $a_{n+1} \in a_{n} R \theta^{t_{n}}\left(a_{n}\right)$ for all $n \geq 0$, there is an integer $m$ such that $a_{m}=0$. We will prove that $P_{\theta}(R)$ is the set of all strongly $\theta$-nilpotent elements in $R$.

Lemma 1.9. Let $P$ be a $\theta$-prime ideal of $R$. If $a \in R \backslash P$, then for any integer $n$ there exists an integer $t_{n} \geq n$ such that $a R \theta^{t_{n}}(a) \nsubseteq P$.

Proof. Since $P$ is $\theta$-invariant and $a \notin P$, we have $\theta^{m}(a) \notin P$ for each integer $m$. For a fixed integer $n$, let $A_{n}=\sum_{k=n}^{\infty} R \theta^{k}(a) R$, then $A_{n}$ is a $\theta$-ideal of $R$ and $(R a R) A_{n} \not \subseteq P$ since $P$ is $\theta$-prime. Now we get $(R a R) A_{n}=\sum_{k=n}^{\infty} R a R \theta^{k}(a) R$, hence $a R \theta^{t_{n}}(a) \nsubseteq \subseteq P$ for some $t_{n} \geq n$.

Theorem 1.10. $P_{\theta}(R)$ is the set of all strongly $\theta$-nilpotent elements in $R$.

Proof. Suppose $a \in P_{\theta}(R)$, then $a x \in P(R[x ; \theta])$ by Lemma 1.2. So $a x$ is strongly nilpotent in $R[x ; \theta]$ by [2, Proposition 3.2.1]. Let $\left(a_{n}\right)_{n=0}^{\infty}$ be a sequence in $R$ such that $a_{0}=a, a_{n+1}=a_{n} r_{n} \theta^{t_{n}}\left(a_{n}\right)$, where $r_{n} \in R$ and $t_{n}$ is a positive integer satisfying $t_{n+1} \geq 1+\sum_{i=0}^{n} t_{i}$ for all $n \geq 0$. For convenience, let $s_{0}=1$, $s_{n}=1+\sum_{i=0}^{n-1} t_{i}, y_{0}=a x$ and $y_{n}=a_{n} x^{s_{n}}$ for all $n \geq 1$. Then $s_{n+1}=s_{n}+t_{n}$, $s_{n} \leq t_{n}$ and hence we have

$$
\begin{aligned}
y_{n+1} & =a_{n+1} x^{s_{n+1}}=a_{n} r_{n} \theta^{t_{n}}\left(a_{n}\right) x^{t_{n}} x^{s_{n}}=a_{n} x^{s_{n}} \theta^{-s_{n}}\left(r_{n}\right) x^{t_{n}-s_{n}} a_{n} x^{s_{n}} \\
& =y_{n} z_{n} y_{n} \in y_{n} R[x ; \theta] y_{n},
\end{aligned}
$$

where $z_{n}=\theta^{-s_{n}}\left(r_{n}\right) x^{t_{n}-s_{n}}$ for all $n \geq 0$. Since $y_{0}=a x$ is strongly nilpotent in $R[x ; \theta], y_{n}=0$ eventually and so does $a_{n}=0$, proving that $a$ is strongly $\theta$-nilpotent.

Conversely let $a \notin P_{\theta}(R)$, then $a \notin P$ for some $\theta$-prime ideal $P$ of $R$. Thus by Lemma 1.8 there is an integer $t_{0} \geq 1$ and $r_{0} \in R$ with $a_{0} r_{0} \theta^{t_{0}}\left(a_{0}\right) \notin P$. Let $a_{1}=a_{0} r_{0} \theta^{t_{0}}\left(a_{0}\right)$, then we get $a_{2}=a_{1} r_{1} \theta^{t_{1}}\left(a_{1}\right) \notin P$ for $r_{1} \in R$ and 
$t_{1} \geq 1+t_{0}$ by applying Lemma 1.8 to $a_{1}$. Repeating this process, we obtain sequences $\left(r_{n}\right)_{n=0}^{\infty},\left(a_{n}\right)_{n=0}^{\infty}$ in $R$ and $\left(t_{n}\right)_{n=0}^{\infty}$ of positive integers such that $t_{n+1} \geq 1+\sum_{i=0}^{n} t_{i}$ and $a_{0}=a, a_{n+1}=a_{n} r_{n} \theta^{t_{n}}\left(a_{n}\right)$ with $a_{n} \notin P$ for all $n \geq 0$. This shows that $a$ is not strongly $\theta$-nilpotent.

Since $\theta^{-1}$ is also an automorphism of $R$, we can define $\theta^{-1}$-primeness and $\theta^{-1}$-semiprimeness analogously. In general $P_{\theta^{-1}}(R)$ need not coincide with $P_{\theta}(R)$ by Example 3.13 below. But Pearson, Stephenson, and Watters [5] gave an affirmative answer for positive powers of $\theta$ as in the following.

Lemma 1.11 ([5, Proposition 4.9]). $P_{\theta^{n}}(R)=P_{\theta}(R)$ for any positive integer $n$; that is, $a \in R$ is strongly $\theta$-nilpotent if and only if a is strongly $\theta^{n}$-nilpotent.

Remark 1.12. In [3] Lam, Leroy, and Matczuk defined the notion of strongly $\theta$ nilpotency and $\theta$-prime radical $\operatorname{rad}(R ; \theta)$ to discuss the prime radicals of $R[x ; \theta]$ and $R\left[x, x^{-1} ; \theta\right]$. But the notions in [3] are different from ours. In Section 3 we will prove that

$$
\operatorname{rad}(R ; \theta)=P(R) \cap P_{\theta}(R) \cap P_{\theta^{-1}}(R) .
$$

Lam, Leroy and Matczuk [3, Definition 3.1(b)] introduce the notion of $\theta$ nilpotency as follows. An element $a$ in $R$ is $\theta$-nilpotent if for each integer $k \geq 1$ there exists an integer $n=n(k) \geq 1$ such that $a \theta^{k}(a) \theta^{2 k}(a) \cdots \theta^{n k}(a)=0$. A $\theta$-invariant ideal $I$ of $R$ is said to be $\theta$-nil if every element in $I$ is $\theta$-nilpotent.

Proposition 1.13. $P_{\theta}(R)$ is $\theta$-nil.

Proof. It is obvious that $P_{\theta}(R)$ is $\theta$-invariant. Thus it suffices to prove that every strongly $\theta$-nilpotent element is $\theta$-nilpotent. Let $a$ be strongly $\theta$-nilpotent and $k \geq 1$. Put $t_{n}=2^{n} k$. Then $t_{0}=k \geq 1$ and $t_{n+1}=2^{n+1} k \geq 1+(1+2+\cdots+$ $\left.2^{n}\right) k=1+\sum_{i=0}^{n} t_{i}$ for all $n \geq 0$. Also let $a_{0}=a$ and $a_{n+1}=a_{n} \theta^{t_{n}}\left(a_{n}\right)$; then $a_{n+1} \in a_{n} R \theta^{t_{n}}\left(a_{n}\right)$. Thus $a_{n}=0$ for some $n \geq 1$ because $a_{0}=a$ is strongly $\theta$-nilpotent. Consequently $0=a_{n}=a \theta^{k}(a) \theta^{2 k}(a) \cdots \theta^{\left(2^{n}-1\right) k}(a)$, entailing that $a$ is $\theta$-nilpotent.

Lemma 1.14 ([3, Theorem 3.5]). Every ring $R$ contains the largest $\theta$-nil ideal, written by $N_{\theta}(R)$, such that $N_{\bar{\theta}}\left(R / N_{\theta}(R)\right)=0$, where $\bar{\theta}$ is the induced automorphism of $R / N_{\theta}(R)$ defined as in Remark 1.4.

The ideal $N_{\theta}(R)$ in Lemma 1.13 is called the $\theta$-nil radical of $R$.

\section{Relations between $P(R)$ and $P_{\theta}(R)$}

In this section we first give some examples of $\theta$ and $R$ and next consider some conditions under which $P(R)$ and $P_{\theta}(R)$ are equal.

Example 2.1. Let $F$ be a field and $A=F\left\{x_{i} \mid i \in \mathbb{Z}\right\}$ be the free algebra with noncommuting indeterminates $\left\{x_{i} \mid i \in \mathbb{Z}\right\}$ over $F$. Let $I$ be the ideal of $A$ generated by the subset $\left\{u^{2} \mid u \in \sum_{i \in \mathbb{Z}} F x_{i}\right\}$ and set $R=A / I$. Then $R$ is the exterior algebra on the set $\left\{\bar{x}_{i} \mid i \in \mathbb{Z}\right\}$, where $\bar{x}_{i}=x_{i}+I$. Let $\theta: R \rightarrow R$ be 
the $F$-automorphism of $R$ induced by the assignment $\bar{x}_{i} \rightarrow \bar{x}_{i+1}$ for all $i \in \mathbb{Z}$. Then clearly $P(R)=\sum_{i \in \mathbb{Z}} R \bar{x}_{i}$. However $P_{\theta}(R)=0$ since $R$ has no nonzero strongly $\theta$-nilpotent elements. In this case $P_{\theta}(R) \varsubsetneqq P(R)$.

Example 2.2. Let $F$ be a field and $B=\prod_{i \in \mathbb{Z}} F_{i}$ with $F_{i}=F$ for all $i$. Let $R$ be the $F$-subalgebra of $B$ generated by $\oplus_{i \in \mathbb{Z}} F_{i}$ and $1_{B}$. For each $i$ set $e_{i}$ to be the idempotent of $B$ such that $e_{i}(j)=\delta_{i j} 1_{F}$, where $\delta_{i j}$ is the Kronecker delta. Let $\theta: R \rightarrow R$ be the automorphism of $R$ induced by the assignment $e_{i} \mapsto e_{i+1}$ for each $i$. Since $R$ is a reduced ring, we have $P(R)=0$. But each $e_{i}$ is strongly $\theta$-nilpotent (also $\theta^{-1}$-nilpotent); hence $P_{\theta}(R)=P_{\theta^{-1}}(R)=\oplus_{i \in \mathbb{Z}} F_{i}$. In this case $P(R) \varsubsetneqq P_{\theta}(R)$.

Example 2.3. Let $R_{1}, \theta_{1}$ be the ring and automorphism respectively as in Example 2.1; and $R_{2}$ and $\theta_{2}$ be the ring and automorphism respectively as in Example 2.2. Set $R=R_{1} \oplus R_{2}$ and define $\theta=\theta_{1} \oplus \theta_{2}$ by $\theta\left(a_{1}, a_{2}\right)=$ $\left(\theta_{1}\left(a_{1}\right), \theta_{2}\left(a_{2}\right)\right)$. Then clearly $\theta$ is an automorphism of $R$, and we have $P(R)=$ $P\left(R_{1}\right) \oplus P\left(R_{2}\right)=P\left(R_{1}\right)$ and $P_{\theta}(R)=P_{\theta_{1}}\left(R_{1}\right) \oplus P_{\theta_{2}}\left(R_{2}\right)=P_{\theta_{2}}\left(R_{2}\right)$ by Examples 2.1 and 2.2. Thus $P(R)$ and $P_{\theta}(R)$ are not comparable.

In Examples 2.1, 2.2, and 2.3, we have $P(R) \neq P_{\theta}(R)$. But $P(R)$ and $P_{\theta}(R)$ are equal under some ascending chain condition as follows.

Note. If $P$ is a $\theta$-semiprime ideal and $A$ is a $\theta^{-1}$-ideal of $R$ such that $A^{2} \subseteq P$, then $A \subseteq P$. In fact, note that $A \theta^{k}(A)=\theta^{k}\left(\theta^{-k}(A) A\right) \subseteq \theta^{k}\left(A^{2}\right) \subseteq \theta^{k}(P)=P$ for any integer $k \geq 0$. Since $P$ is $\theta$-semiprime, we have $A \subseteq P$.

Proposition 2.4. If $R$ satisfies the ascending chain condition on $\theta$-ideals, then $P(R)=P_{\theta}(R)$ and especially $P(R[x ; \theta])=P(R)[x ; \theta]$.

Proof. First note that if $R$ satisfies the ascending chain condition on $\theta$-ideals, then every $\theta$-ideal is $\theta$-invariant. Suppose that $A$ is an ideal of $R$ and $m$ is an integer such that $A \theta^{k}(A) \subseteq P(R)$ for all $k \geq m$. Then $A B \subseteq P(R)$ with $B=\sum_{k=m}^{\infty} \theta^{k}(A)$. Since $B$ is a $\theta$-ideal, $B$ is $\theta$-invariant. If $P$ is any prime ideal of $R$, then $A B \subseteq P(R) \subseteq P$ and so $A \subseteq P$ or $B \subseteq P$. If $B \subseteq P$ then $A \subseteq \theta^{-m}(B)=B \subseteq P$. In any case $A \subseteq P$, entailing $A \subseteq P(R)$. This proves $P_{\theta}(R) \subseteq P(R)$ by Corollary 1.7 .

For the converse inclusion, it is enough to show that $P_{\theta}(R)$ is a semiprime ideal of $R$. Suppose that $I$ is an ideal of $R$ such that $I^{2} \subseteq P_{\theta}(R)$. Then clearly $\left(\theta^{k}(I)\right)^{2}=\theta^{k}\left(I^{2}\right) \subseteq \theta^{k}\left(P_{\theta}(R)\right)=P_{\theta}(R)$ for any integer $k$. Choose any element $a \in I$ and let $C=\sum_{k=0}^{\infty} R \theta^{k}(a) R$. Then $C$ is a $\theta$-ideal of $R$ and hence is $\theta$-invariant, entailing $a \in C=\theta(C)=\sum_{k=1}^{\infty} R \theta^{k}(a) R$. This implies that $a \in R \theta(a) R+\cdots+R \theta^{m}(a) R$ for some $m \geq 1$. Let $D=\sum_{k=1}^{m} R \theta^{k}(a) R$; then $D$ is a $\theta^{-1}$-ideal such that

$$
D^{m+1}=\left(\sum_{k=1}^{m} R \theta^{k}(a) R\right)^{m+1} \subseteq \sum_{k=1}^{m}\left(R \theta^{k}(a) R\right)^{2} \subseteq \sum_{k=1}^{m}\left(\theta^{k}(I)\right)^{2} \subseteq P_{\theta}(R) .
$$

By the preceding Note combined with induction on $m \geq 1$, we have $D \subseteq P_{\theta}(R)$. Since $a \in D, a \in P_{\theta}(R)$ and hence $I \subseteq P_{\theta}(R)$. 
Corollary 2.5. If $R$ is left or right Noetherian, then $P_{\theta}(R)=P_{\theta^{-1}}(R)=P(R)$ for any automorphism $\theta$ of $R$.

An automorphism $\theta$ of $R$ is called of locally finite order if for any $a \in R$ there is an integer $n=n(a) \geq 1$ such that $\theta^{n}(a)=a$. Bedi and Ram proved that if $\theta$ is of locally finite order, then the Jacobson radicals of $R[x ; \theta]$ and $R\left[x, x^{-1} ; \theta\right]$ have much nicer forms [1, Corollary 3.3 and Theorem 3.7]. We also prove that if $\theta$ is of locally finite order, then $P_{\theta}(R)=P(R)$ in the following.

Proposition 2.6. If $\theta$ is of locally finite order, then $P(R)=P_{\theta}(R)=P_{\theta^{-1}}(R)$.

Proof. It suffices to prove that $P(R)$ is $\theta$-semiprime and $P_{\theta}(R)$ is semiprime. Let $I$ be an ideal of $R$ and $m$ be an integer such that $I \theta^{k}(I) \subseteq P(R)$ for all $k \geq m$. Set $A=R a R$ for $a \in I$. Since $\theta$ is of locally finite order there is an integer $n \geq 1$ such that $\theta^{n}(a)=a$. Thus $\theta^{n k}(a)=a$ for any integer $k$. Choose a positive integer $k$ such that $k \geq m$, then $n k \geq m$ and hence we have $A^{2}=A \theta^{n k}(A) \subseteq I \theta^{n k}(I) \subseteq P(R)$. Thus we obtain $A \subseteq P(R)$ and $a \in P(R)$, proving that $I \subseteq P(R)$ and $P(R)$ is $\theta$-semiprime.

To show that $P_{\theta}(R)$ is semiprime, let $J$ be an ideal of $R$ with $J^{2} \subseteq P_{\theta}(R)$. Since $P_{\theta}(R)$ is $\theta$-invariant, $\theta^{k}\left(J^{2}\right) \subseteq \theta^{k}\left(P_{\theta}(R)\right)=P_{\theta}(R)$ for each integer $k$. Let $b \in J$ and choose an integer $n \geq 1$ such that $\theta^{n}(b)=b$. Set $B=\sum_{k=0}^{n-1} R \theta^{k}(b) R$. Then $B$ is a $\theta$-ideal of $R$ and $B^{n+1} \subseteq \sum_{k=0}^{n-1}\left(R \theta^{k}(b) R\right)^{2} \subseteq \sum_{k=0}^{n-1} \theta^{k}\left(J^{2}\right) \subseteq$ $P_{\theta}(R)$. Since $P_{\theta}(R)$ is $\theta$-semiprime and $B$ is a $\theta$-ideal in $R$ we have $b \in B \subseteq$ $P_{\theta}(R)$, entailing $J \subseteq P_{\theta}(R)$. Thus $P_{\theta}(R)$ is a semiprime ideal of $R$.

An important class of automorphisms is the class of power-quasi-inner ones. According to Pearson et al. [5], an automorphism $\theta$ of $R$ is called quasi-inner ( $Q I$ for short) if there exists a regular element (i.e., neither left nor right zerodivisor) $u \in R$ such that $u r=\theta(r) u$ for all $r \in R$, and $\theta$ is called power-quasiinner ( $P Q I$ for short) if $\theta^{n}$ is QI for some positive integer $n$.

Remark 2.7. Let $\theta$ be a PQI automorphism of $R$. Then there are an integer $n \geq 1$ and a regular element $u \in R$ such that $u r=\theta^{n}(r) u$ for all $r \in R$. We call such a regular element $u$ an axis for $\theta$. If $u$ is an axis for $\theta$, then $R u=u R$ (hence this is a two-sided ideal of $R$ ). Moreover if $u$ is an axis for $\theta$, then $u^{n}$ is also an axis for $\theta$ for all $n \geq 1$. Pearson, Stephenson and Watters proved that if $\theta$ is PQI, then there is an axis $u$ for $\theta$ such that $\theta(u)=u$ [5, Proposition 4.7].

We will find relations among $P(R), P_{\theta}(R)$ and $P_{\theta^{-1}}(R)$ for a PQI automorphism $\theta$ of $R$. First notice the following lemma, obtained from [5, Lemma 4.11].

Lemma 2.8. Suppose that $\theta$ is a PQI automorphism of $R$ and $u \in R$ is an axis for $\theta$. Then $P(R) u \subseteq P_{\theta}(R)$ and $P_{\theta}(R) u \subseteq P(R)$.

Lemma 2.9. Let $\theta$ be a $Q I$ automorphism of $R$ with an axis $u$ satisfying $u r=\theta(r) u$ for all $r \in R$. Then

(1) If $a \in R$ with $a u \in P(R)$, then $a \in P_{\theta}(R)$. 
(2) If $a \in R$ with $a u \in P_{\theta^{-1}}(R)$, then $a \in P(R)$.

Proof. The proofs are similar to that of Theorem 1.9. Since $\theta$ is QI, $\theta(u)=u$ by definition.

(1) If $a u \in P(R)$, then $a u$ is strongly nilpotent in $R$. It suffices to show that $a$ is strongly $\theta$-nilpotent by Theorem 1.9 . Let $\left(a_{n}\right)_{n=0}^{\infty}$ be a sequence in $R$ such that $a_{0}=a$ and $a_{n+1}=a_{n} r_{n} \theta^{t_{n}}\left(a_{n}\right)$, where $r_{n} \in R$ and $t_{0} \geq 1$, $t_{n+1} \geq 1+\sum_{i=0}^{n} t_{i}$ for all $n \geq 0$. For convenience let $s_{0}=1, s_{n+1}=1+\sum_{i=0}^{n} t_{i}$. Then $s_{n+1}=s_{n}+t_{n}$ and $s_{n} \leq t_{n}<s_{n+1}$. Letting $b_{n}=a_{n} u^{s_{n}}$ for all $n \geq 0$, then we have $b_{0}=a u$ and

$$
\begin{aligned}
b_{n+1} & =a_{n+1} u^{s_{n+1}}=a_{n} r_{n} \theta^{t_{n}}\left(a_{n}\right) u^{t_{n}} u^{s_{n}} \\
& =a_{n} u^{s_{n}} \theta^{-s_{n}}\left(r_{n}\right) u^{t_{n}-s_{n}} a_{n} u^{s_{n}}=b_{n}\left(\theta^{-s_{n}}\left(r_{n}\right) u^{t_{n}-s_{n}}\right) b_{n} \in b_{n} R b_{n}
\end{aligned}
$$

for all $n \geq 0$. Since $b_{0}=a u$ is strongly nilpotent, $b_{m}=0$ for some $m \geq 0$, causing $a_{m}=0$ because $u$ is regular. Thus $a$ is strongly $\theta$-nilpotent.

(2) If $a u \in P_{\theta^{-1}}(R)$, then $a u$ is strongly $\theta^{-1}$-nilpotent. We will show that $a$ is strongly nilpotent. Let $\left(c_{n}\right)_{n=0}^{\infty}$ be a sequence in $R$ such that $c_{0}=a$ and $c_{n+1}=c_{n} r_{n} c_{n}$, where $r_{n} \in R$ for all $n \geq 0$. Let $t_{n}=2^{n}$, then clearly $t_{0} \geq 1$, $t_{n+1} \geq 1+\sum_{i=0}^{n} t_{i}$ for all $n \geq 0$. Put $d_{n}=c_{n} u^{t_{n}}=c_{n} u^{2^{n}}$. Then we have $d_{0}=c_{0} u=a u$ and

$$
\begin{aligned}
d_{n+1} & =c_{n+1} u^{t_{n+1}}=c_{n} r_{n} c_{n} u^{2^{n+1}}=c_{n} r_{n} c_{n} u^{2^{n}} u^{2^{n}} \\
& =c_{n} u^{2^{n}} \theta^{-2^{n}}\left(r_{n}\right) \theta^{-2^{n}}\left(c_{n} u^{2^{n}}\right)=d_{n} \theta^{-2^{n}}\left(r_{n}\right) \theta^{-t_{n}}\left(d_{n}\right) \in d_{n} R \theta^{-t_{n}}\left(d_{n}\right),
\end{aligned}
$$

for all $n \geq 0$. Since $d_{0}=a u$ is strongly $\theta^{-1}$-nilpotent, we get $d_{k}=0$ for some $k \geq 1$, causing $c_{k}=0$ because $u$ is regular. Thus $a$ is strongly nilpotent.

Note. In Lemma 2.9 we also obtain that $u a \in P(R)$ (resp. $u a \in P_{\theta^{-1}}(R)$ ) implies $a \in P_{\theta}(R)$ (resp. $a \in P(R)$ ), by similar proofs.

Lemma 2.10. If $\theta$ is a PQI automorphism of $R$, then we have the following assertions:

(1) $P_{\theta^{-1}}(R) \subseteq P(R) \subseteq P_{\theta}(R)$;

(2) Every axis $u$ for $\theta$ is regular modulo $P_{\theta}(R)$.

Proof. (1) Suppose that $\theta^{n}$ is QI for some $n \geq 1$, then $P_{\theta^{-n}}(R) \subseteq P(R) \subseteq$ $P_{\theta^{n}}(R)$ by Lemma 2.9. But $P_{\theta^{n}}(R)=P_{\theta}(R)$ and $P_{\theta^{-n}}(R)=P_{\theta^{-1}}(R)$ by Lemma 1.10; hence we have $P_{\theta^{-1}}(R) \subseteq P(R) \subseteq P_{\theta}(R)$.

(2) Suppose that $u$ is an axis for $\theta$ and $a \in R$ with $a u \in P_{\theta}(R)$. Then $a u^{2} \in P(R)$ by Lemma 2.8. Since $u^{2}$ is also an axis for $\theta$, we have $a \in P_{\theta}(R)$ by Lemma 2.9(1) and Lemma 1.10. We also obtain that $u a \in P_{\theta}(R)$ implies $a \in P_{\theta}(R)$ by Note of Lemma 2.9. Thus $u$ is regular modulo $P_{\theta}(R)$.

The class of QI automorphisms is large as can be seen by the following construction. 
Example 2.11. Let $\theta$ be any automorphism of $R$ and $S=R[x ; \theta]$. Define $\theta^{*}: S \rightarrow S$ by $\theta^{*}\left(\sum_{i} a_{i} x^{i}\right)=\sum_{i} \theta\left(a_{i}\right) x^{i}$. Then $\theta^{*}$ is a QI automorphism of $S$ with an axis $x$, i.e., $x f(x)=\theta^{*}(f(x)) x$ for all $f(x) \in S$.

Corollary 2.12. Let $S=R[x ; \theta]$ and $\sigma=\theta^{*}$ be as in Example 2.11. Then $P_{\sigma^{-1}}(S) \subseteq P(S) \subseteq P_{\sigma}(S)$

Proof. By Lemma 2.10(1) and Example 2.11.

Note. For any automorphism $\theta$ of $R$ we have $P(S)=\left(P(R) \cap P_{\theta}(R)\right)+$ $\sum_{i \geq 1} P_{\theta}(R) x^{i}$ by Lemma 1.2 , where $S=R[x ; \theta]$. So we also obtain $P_{\sigma}(S)=$ $\sum_{i>0} P_{\theta}(R) x^{i}=P_{\theta}(R)[x ; \theta]$ with the help of Lemma 2.10(2), where $\sigma=\theta^{*}$ as in Corollary 2.12. Therefore $P(S)=P_{\sigma}(S)$ if and only if $P_{\theta}(R) \subseteq P(R)$.

In Section 3 we will show $P_{\sigma^{-1}}(S)=\left(P(R) \cap P_{\theta}(R) \cap P_{\theta^{-1}}(R)\right)[x ; \theta]$ and also give an example of $R$ with a QI automorphism $\theta$ such that $P_{\theta^{-1}}(R) \varsubsetneqq P(R) \varsubsetneqq$ $P_{\theta}(R)$. But we have the following equality.

Proposition 2.13. Let $\theta$ be a PQI automorphism of $R$. Then the following conditions are equivalent:

(1) $P(R)=P_{\theta}(R)$;

(2) Every axis $u$ for $\theta$ is regular modulo $P(R)$;

(3) Some axis $u$ for $\theta$ is regular modulo $P(R)$.

Proof. $(1) \Rightarrow(2)$ follows directly from Lemma $2.10(2)$ and $(2) \Rightarrow(3)$ is obvious. To prove $(3) \Rightarrow(1)$, let $u$ be an axis for $\theta$ which is regular modulo $P(R)$. If $a \in P_{\theta}(R)$, then $a u \in P(R)$ by Lemma 2.8, forcing $a \in P(R)$ since $u$ is regular modulo $P(R)$. Thus we have $P(R)=P_{\theta}(R)$ with the help of Lemma 2.10(1).

Corollary 2.14. Let $\theta$ be a PQI automorphism of $R$. If there is an axis $u$ for $\theta$ which is regular modulo $P(R)$, then $P(R[x ; \theta])=P(R)[x ; \theta]$.

Proof. By Proposition 2.13 and Note after Corollary 2.12.

\section{The prime radical of $R\left[x, x^{-1} ; \theta\right]$}

Let $\theta$ be an automorphism of $R$. We use $S$ and $T$ to denote $R[x ; \theta]$ and $R\left[x, x^{-1} ; \theta\right]$ respectively, where $R\left[x, x^{-1} ; \theta\right]$ is the skew Laurent polynomial ring with an indeterminate $x$ over $R$. Let $\sigma=\theta^{*}$ be the automorphism of $S$ defined by $\sigma\left(\sum_{i \geq 0} a_{i} x^{i}\right)=\sum_{i \geq 0} \theta\left(a_{i}\right) x^{i}$. Then $\sigma$ is QI with an axis $x$. Note that $T=$ $S X^{-1}$, the (right) quotient ring of $S$ by the set $X=\left\{x^{n} \mid n \in \mathbb{Z}\right.$ with $\left.n \geq 0\right\}$.

In this section we will prove $P(T)=P_{\sigma^{-1}}(S) X^{-1}=\left(P(R) \cap P_{\theta}(R) \cap\right.$ $\left.P_{\theta^{-1}}(R)\right)\left[x, x^{-1} ; \theta\right]$ and characterize elements in $P(T)$. The following lemma is obvious.

Lemma 3.1. Let $K$ be a proper ideal of $T$. Then we have the following assertions:

(1) $K \cap S=\left\{f(x) \in S \mid f(x) x^{-m} \in K\right.$ for some integer $\left.m \geq 0\right\}$. 
(2) $K \cap S$ is a $\sigma$-invariant ideal of $S$ and $(K \cap S) X^{-1}=K$.

(3) $x \notin K \cap S$ and $x$ is regular modulo $K \cap S$.

Now we need some technical definitions. A proper $\theta$-invariant ideal $P$ of $R$ is said to be $\left(\theta, \theta^{-1}\right)$-prime provided that $A \subseteq P$ or $B \subseteq P$ whenever $A B \subseteq P$ for $\theta$-invariant ideals $A, B$ in $R$. A proper $\theta$-invariant ideal $Q$ of $R$ is said to be $\left(\theta, \theta^{-1}\right)$-semiprime provided that if $A$ is a $\theta$-invariant ideal of $R$ with $A^{2} \subseteq Q$ then $A \subseteq Q$. Clearly every $\theta$-prime and every $\theta^{-1}$-prime (resp. every $\theta$-semiprime and every $\theta^{-1}$-semiprime) ideal is $\left(\theta, \theta^{-1}\right)$-prime $\left(\operatorname{resp} .\left(\theta, \theta^{-1}\right)\right.$ semiprime). Observe that $P(R), P_{\theta}(R)$ and $P_{\theta^{-1}}(R)$ are all $\left(\theta, \theta^{-1}\right)$-semiprime. Also note that an intersection of any set of $\left(\theta, \theta^{-1}\right)$-semiprime ideals is $\left(\theta, \theta^{-1}\right)$ semiprime. In particular the intersection of all the $\left(\theta, \theta^{-1}\right)$-semiprime ideals of $R$ is $\left(\theta, \theta^{-1}\right)$-semiprime, and hence $R$ contains the smallest $\left(\theta, \theta^{-1}\right)$-semiprime ideal. As in the classical case, we define the $\left(\theta, \theta^{-1}\right)$-prime radical $P_{\left(\theta, \theta^{-1}\right)}(R)$ by

$$
P_{\left(\theta, \theta^{-1}\right)}(R)=\bigcap\left\{P \mid P \text { is a }\left(\theta, \theta^{-1}\right) \text {-prime ideal of } R\right\} .
$$

Notice that an ideal $I$ of $R$ is $\theta$-invariant if and only if $I[x ; \theta]=\left\{\sum_{i \geq 0} a_{i} x^{i}\right.$ $a_{i} \in I$ for all $\left.i\right\}$ is a $\sigma$-invariant ideal of $S$ if and only if the set $I\left[x, x^{-1} ; \theta\right]=$ $\left\{\sum_{i=-m}^{n} a_{i} x^{i} \mid m, n \geq 0, a_{i} \in I\right.$ for all $\left.i\right\}$ is an ideal of $T$.

Proposition 3.2. Let $I$ be a proper $\theta$-invariant ideal of $R$. Then we have the following assertions:

(1) $I$ is $\left(\theta, \theta^{-1}\right)$-prime if and only if $I\left[x, x^{-1} ; \theta\right]$ is a prime ideal of $T$.

(2) $I$ is $\left(\theta, \theta^{-1}\right)$-semiprime if and only if $I\left[x, x^{-1} ; \theta\right]$ is a semiprime ideal of $T$.

Proof. (1) Suppose that $I$ is a $\left(\theta, \theta^{-1}\right)$-prime ideal of $R$ and let $H, K$ be ideals of $T$ such that $H K \subseteq I\left[x, x^{-1} ; \theta\right]$. Assume on the contrary that $H \nsubseteq I\left[x, x^{-1} ; \theta\right]$ and $K \nsubseteq I\left[x, x^{-1} ; \theta\right]$. Then by Lemma 3.1 , there are polynomials $f(x)=$ $\sum_{i=0}^{m} a_{i} x^{i} \in H \cap S$ and $g(x)=\sum_{j=0}^{n} b_{j} x^{j} \in K \cap S$ such that $f(x) \notin I\left[x, x^{-1} ; \theta\right]$ and $g(x) \notin I\left[x, x^{-1} ; \theta\right]$. Let $p$ and $q$ be the first integers such that $a_{p} \notin I$ and $b_{q} \notin I$, respectively. Then for any integer $k \in \mathbb{Z}$ and any $r \in R$, the coefficient of $x^{p+q}$ in $\sigma^{k}(f(x)) r g(x)$ is

$$
\sum_{i=0}^{p+q} \theta^{k}\left(a_{i}\right) \theta^{i}(r) \theta^{i}\left(b_{p+q-i}\right) \in I .
$$

Since $\theta^{k}\left(a_{i}\right) \in I$ and $b_{j} \in I$ for all $i, j$ with $0 \leq i<p, 0 \leq j<q$, we have $\theta^{k}\left(a_{p}\right) \theta^{p}(r) \theta^{p}\left(b_{q}\right) \in I$ for all $k \in \mathbb{Z}, r \in R$. Thus for any integers $k$ and $l$ we get

$$
\begin{gathered}
\theta^{k}\left(a_{p}\right) R \theta^{l}\left(b_{q}\right)=\theta^{l-p}\left(\theta^{k-l+p}\left(a_{p}\right) R \theta^{p}\left(b_{q}\right)\right) \subseteq I, \\
\left(\sum_{k=-\infty}^{\infty} R \theta^{k}\left(a_{p}\right) R\right)\left(\sum_{k=-\infty}^{\infty} R \theta^{l}\left(b_{q}\right) R\right) \subseteq I .
\end{gathered}
$$


But $\sum_{k=-\infty}^{\infty} R \theta^{k}\left(a_{p}\right) R, \sum_{k=-\infty}^{\infty} R \theta^{l}\left(b_{q}\right) R$ are $\theta$-invariant and $I$ is $\left(\theta, \theta^{-1}\right)$ prime, we obtain that $\sum_{k=-\infty}^{\infty} R \theta^{k}\left(a_{p}\right) R \subseteq I$ or $\sum_{k=-\infty}^{\infty} R \theta^{l}\left(b_{q}\right) R \subseteq I$. Consequently $a_{p} \in I$ or $b_{q} \in I$, a contradiction to the choice of $p$ and $q$. Therefore $I\left[x, x^{-1} ; \theta\right]$ is a prime ideal of $T$.

Conversely suppose that $I\left[x, x^{-1} ; \theta\right]$ is a prime ideal of $T$ and that $A, B$ are $\theta$-invariant ideals of $R$ such that $A B \subseteq I$. Then $A\left[x, x^{-1} ; \theta\right] B\left[x, x^{-1} ; \theta\right]=$ $A B\left[x, x^{-1} ; \theta\right] \subseteq I\left[x, x^{-1} ; \theta\right]$ and hence $A\left[x, x^{-1} ; \theta\right] \subseteq I\left[x, x^{-1} ; \theta\right]$ or $B\left[x, x^{-1} ; \theta\right]$ $\subseteq I\left[x, x^{-1} ; \theta\right]$, forcing $A \subseteq I$ or $B \subseteq I$. Thus $I$ is $\left(\theta, \theta^{-1}\right)$-prime.

(2) The case of semiprimeness can be proved by taking $H=K$ and $A=B$ in the proof of (1).

Lemma 3.3. Let $H$ be a $\sigma$-invariant ideal of $S$ such that $x \notin H$ and $x$ is regular modulo $H$. If $A$ and $B$ are ideals of $S$ such that $A B \subseteq H$ and $\sigma^{-1}(B) \subseteq B$, then there exist $\sigma$-invariant (hence $\sigma^{-1}$-invariant) ideals $C$ and $D$ such that $A \subseteq C, B \subseteq D$ and $C D \subseteq H$.

Proof. Note that $A \sigma^{i}(B) x^{i}=A x^{i} B \subseteq A B \subseteq H$ for any integer $i \geq 0$, and so $A \sigma^{i}(B) \subseteq H$ since $x$ is regular modulo $H$. Let $D=\sum_{i=0}^{\infty} \sigma^{i}(B)$. Then $D$ is a $\sigma$-invariant ideal with $B \subseteq D$ and $A D \subseteq H$. Moreover $\sigma^{j}(A) D=\sigma^{j}(A D) \subseteq$ $\sigma^{j}(H)=H$ for each integer $j$. Thus if we let $C=\sum_{i=-\infty}^{\infty} \sigma^{j}(A)$, then $C$ is $\sigma$-invariant and $A \subseteq C, C D \subseteq H$.

Proposition 3.4. Let $I$ be a proper $\theta$-invariant ideal of $R$.

(1) $I$ is $\left(\theta, \theta^{-1}\right)$-prime if and only if $I[x ; \theta]$ is a $\sigma^{-1}$-prime ideal of $S$. $S$.

(2) $I$ is $\left(\theta, \theta^{-1}\right)$-semiprime if and only if $I[x ; \theta]$ is a $\sigma^{-1}$-semiprime ideal of

Proof. (1) Suppose that $I$ is $\left(\theta, \theta^{-1}\right)$-prime and $C, D$ are ideals of $S$ with $C D \subseteq$ $I[x ; \theta]$ and $\sigma^{-1}(D) \subseteq D$. By Lemma 3.3 we can assume that $C, D$ are $\sigma$ invariant. If $C \nsubseteq I[x ; \theta]$ and $D \nsubseteq I[x ; \theta]$, then the same argument as in the proof of Proposition 3.2(1) leads to a contradiction. Thus $C \subseteq I[x ; \theta]$ of $D \subseteq I[x ; \theta]$.

Conversely suppose that $I[x ; \theta]$ is $\sigma^{-1}$-prime and $A, B$ are $\theta$-invariant ideals of $R$ such that $A B \subseteq I$. Then $A[x ; \theta]$ and $B[x ; \theta]$ are $\sigma$-invariant ideals of $S$ satisfying $A[x ; \theta] B[x ; \theta]=A B[x ; \theta] \subseteq I[x ; \theta]$, whence $A \subseteq I$ or $B \subseteq I$.

(2) Suppose $I$ is $\left(\theta, \theta^{-1}\right)$-semiprime. Let $C$ be an ideal of $S$ and $m$ be an integer such that $C \sigma^{-k}(C) \subseteq I[x ; \theta]$ for all $k \geq m$. Let $D=\sum_{k \geq m} \sigma^{-k}(C)$, then $D$ is a $\sigma^{-1}$-ideal and $C D \subseteq I[x ; \theta]$. Since $x \notin I[x ; \theta]$ and $x$ is regular modulo $I[x ; \theta]$, we can assume that $C$ and $D$ are $\sigma^{-1}$-invariant by Lemma 3.3. Thus we can also assume without loss of generality that $C$ is $\sigma^{-1}$-invariant and $C^{2} \subseteq I[x ; \theta]$. If $C \nsubseteq I[x ; \theta]$, then a similar argument as in the proof of Proposition $3.2(1)$ leads to a contradiction. So $C \subseteq I[x ; \theta]$, concluding that $I[x ; \theta]$ is $\sigma^{-1}$-semiprime.

Conversely suppose that $I[x ; \theta]$ is $\sigma^{-1}$-semiprime and $A$ is a $\theta$-invariant ideal of $R$ with $A^{2} \subseteq I$. Then $A[x ; \theta]^{2}=A^{2}[x ; \theta] \subseteq I[x ; \theta]$. Since $A[x ; \theta]$ is $\sigma^{-1}$-invariant, $A[x ; \theta] \subseteq I[x ; \theta]$ and $A \subseteq I$. Thus $I$ is $\left(\theta, \theta^{-1}\right)$-semiprime. 
Corollary 3.5. The following conditions are equivalent:

(1) $R$ is $\left(\theta, \theta^{-1}\right)$-prime (resp. $\left(\theta, \theta^{-1}\right)$-semiprime);

(2) $S$ is $\sigma^{-1}$-prime (resp. $\sigma^{-1}$-semiprime);

(3) $T$ is prime (resp. semiprime).

We may compare Corollary 3.5 with [3, Theorem 4.21].

Lemma 3.6. (1) If $P$ is a $\sigma^{-1}$-prime ideal of $S$, then $P \cap R$ is a $\left(\theta, \theta^{-1}\right)$-prime ideal of $R$ and $(P \cap R)[x ; \theta] \subseteq P$.

(2) If $Q$ is a prime ideal of $T$, then $Q \cap S$ is a $\sigma^{-1}$-prime ideal of $S$. In particular $Q \cap R$ is a $\left(\theta, \theta^{-1}\right)$-prime ideal of $R$ with $(Q \cap R)\left[x, x^{-1} ; \theta\right] \subseteq Q$.

Proof. (1) Let $P$ be a $\sigma^{-1}$-prime ideal of $S$. Then $P \cap R$ is $\theta$-invariant and $(P \cap R)[x ; \theta] \subseteq P$ because of $\sigma(P)=P$. Let $A, B$ be $\theta$-invariant ideals of $R$ with $A B \subseteq P \cap R$. Then $A[x ; \theta] B[x ; \theta]=(A B)[x ; \theta] \subseteq(P \cap R)[x ; \theta] \subseteq P$. Since $A[x ; \theta]$ and $B[x ; \theta]$ are $\sigma^{-1}$-ideals of $S$, we have $A[x ; \theta] \subseteq P$ or $B[x ; \theta] \subseteq P$; hence $A \subseteq P \cap R$ or $B \subseteq P \cap R$, showing that $P \cap R$ is $\left(\theta, \theta^{-1}\right)$-prime.

(2) Let $Q$ be a prime ideal of $T$. Then $Q \cap S$ is a $\sigma$-invariant ideal of $S$ with $(Q \cap S) X^{-1}=Q$ by Lemma 3.1. Let $C, D$ be ideals of $S$ with $C D \subseteq Q \cap S$ and $\sigma^{-1}(D) \subseteq D$. Since $x \notin Q \cap S$ and $x$ is regular modulo $Q \cap S$, it follows from Lemma 3.3 that $C$ and $D$ can be assumed $\sigma$-invariant. Thus $C X^{-1}$ and $D X^{-1}$ are ideals of $T$ such that $C X^{-1} D X^{-1}=(C D) X^{-1} \subseteq(Q \cap S) X^{-1}=Q$. Since $Q$ is prime we have that $C X^{-1} \subseteq Q$ or $D X^{-1} \subseteq Q$, yielding $C \subseteq Q \cap S$ or $D \subseteq Q \cap S$. Thus $Q \cap S$ is $\sigma^{-1}$-prime. Now the $\left(\theta, \theta^{-1}\right)$-primeness of $Q \cap R=(Q \cap S) \cap R$ is an immediate consequence of (1).

The following proposition is obtained from Propositions 3.2, 3.4 and Lemma 3.6 .

Proposition 3.7. (1) $P_{\sigma^{-1}}(S)=P_{\left(\theta, \theta^{-1}\right)}(R)[x ; \theta]$.

(2) $P(T)=P_{\left(\theta, \theta^{-1}\right)}(R)\left[x, x^{-1} ; \theta\right]=P_{\sigma^{-1}}(S) X^{-1}$.

Corollary 3.8. $P_{\left(\theta, \theta^{-1}\right)}(R)$ is the smallest $\left(\theta, \theta^{-1}\right)$-semiprime ideal of $R$. Especially $P_{\left(\theta, \theta^{-1}\right)}(R) \subseteq P(R) \cap P_{\theta}(R) \cap P_{\theta^{-1}}(R)$.

To prove $P_{\left(\theta, \theta^{-1}\right)}(R)=P(R) \cap P_{\theta}(R) \cap P_{\theta^{-1}}(R)$ and characterize elements of $P(T)$, we need one more related definition. An element $a$ in $R$ is said to be strongly $\left(\theta, \theta^{-1}\right)$-nilpotent provided that given any sequence $\left(t_{n}\right)_{n=0}^{\infty}$ of integers, every sequence $\left(a_{n}\right)_{n=0}^{\infty}$, such that $a_{0}=a$ and $a_{n+1} \in a_{n} R \theta^{t_{n}}\left(a_{n}\right)$ for all $n \geq 0$, is eventually zero [3, Definition 1.8].

Lemma 3.9. (1) For any $a \in R \backslash P_{\left(\theta, \theta^{-1}\right)}(R)$ there are $r \in R$ and an integer $t$ such that $\operatorname{ar} \theta^{t}(a) \notin P_{\left(\theta, \theta^{-1}\right)}(R)$.

(2) If $a \in R$ is strongly $\left(\theta, \theta^{-1}\right)$-nilpotent, then $a \in P_{\left(\theta, \theta^{-1}\right)}(R)$.

(3) If $a \in P(R) \cap P_{\theta}(R) \cap P_{\theta^{-1}}(R)$, then a is strongly $\left(\theta, \theta^{-1}\right)$-nilpotent.

Proof. (1) Suppose that $a \in R$ and $a R \theta^{i}(a) \subseteq P_{\left(\theta, \theta^{-1}\right)}(R)$ for any integer $i$. Then for all $i, j \in \mathbb{Z}$

$$
\theta^{i}(a) R \theta^{j}(a)=\theta^{i}\left(a R \theta^{j-i}(a)\right) \subseteq \theta^{i}\left(P_{\left(\theta, \theta^{-1}\right)}(R)\right)=P_{\left(\theta, \theta^{-1}\right)}(R) .
$$


Thus if $A=\sum_{i=-\infty}^{\infty} R \theta^{i}(a) R$, then $A^{2} \subseteq P_{\left(\theta, \theta^{-1}\right)}(R)$. Since $A$ is $\theta$-invariant, we have $A \subseteq P_{\left(\theta, \theta^{-1}\right)}(R)$ and $a \in P_{\left(\theta, \theta^{-1}\right)}(R)$.

(2) Let $a \notin P_{\left(\theta, \theta^{-1}\right)}(R)$. Then $a r_{0} \theta^{t_{0}}(a) \notin P_{\left(\theta, \theta^{-1}\right)}(R)$ for some $r_{0} \in R$ and $t_{o} \in \mathbb{Z}$ by (1). Let $a_{1}=\operatorname{ar}_{0} \theta^{t_{0}}(a)$ and apply (1) again to $a_{1}$. Then we get $a_{2}=a_{1} r_{1} \theta^{t_{1}}\left(a_{1}\right) \notin P_{\left(\theta, \theta^{-1}\right)}(R)$ for some $r_{1} \in R$ and $t_{1} \in \mathbb{Z}$. Inductively there exists a sequence $\left(t_{n}\right)_{n=0}^{\infty}$ of integers and a sequence $\left(r_{n}\right)_{n=0}^{\infty}$ in $R$ such that $a_{n} \notin P_{\left(\theta, \theta^{-1}\right)}(R)$ for all $n \in\{0,1,2, \ldots\}$, where $a_{0}=a$ and $a_{k+1}=a_{k} r_{k} \theta^{t_{k}}\left(a_{k}\right)$ for all $k \in\{0,1,2, \ldots\}$. Thus $a$ is not strongly $\left(\theta, \theta^{-1}\right)$-nilpotent.

(3) Let $a \in P(R) \cap P_{\theta}(R) \cap P_{\theta^{-1}}(R)$ and suppose that $\left(t_{n}\right)_{n=0}^{\infty}$ is a sequence in $\mathbb{Z}$ and $\left(r_{n}\right)_{n=0}^{\infty}$ is a sequence in $R$. Next set $a_{0}=a, a_{k+1}=a_{k} r_{k} \theta^{t_{k}}\left(a_{k}\right)$ for all $k \in\{0,1,2, \ldots\}$ and $s_{n}=\sum_{i=0}^{n} t_{i}$ for all $n \geq 0$. Notice that for all $i, j \in \mathbb{Z}$ with $0 \leq i<j$

$$
a_{i+1} \in a R \theta^{s_{i}}(a) \text { and } a_{j+1} \in a_{i+1} R \theta^{\left(s_{j}-s_{i}\right)}\left(a_{i+1}\right) .
$$

In particular if $0 \leq i<j$ and $s_{i}=s_{j}$ then $a_{j+1} \in a_{i+1} R a_{i+1}$. We will show that $a_{n}=0$ for some $n \geq 0$. The proof splits into the following two cases.

Case 1. When the sequence $\left(s_{n}\right)_{n=0}^{\infty}$ is bounded.

Assume that $\left(s_{n}\right)_{n=0}^{\infty}$ is bounded. Then there is an integer $m$ such that $s_{k}=m$ for infinitely many $k$ 's. Choose a sequence $(n(k))_{k=0}^{\infty}$ of positive integers such that $1 \leq n(0)<n(1)<n(2)<\cdots$ and $s_{n(k)}=m$ for all $k \geq 0$. Let $b_{k}=a_{n(k)+1}$ for all $k \geq 0$; then by $(*)$ we have

$b_{k+1}=a_{n(k+1)+1} \in a_{n(k)+1} R \theta^{\left(s_{n(k+1)}-s_{n(k)}\right)}\left(a_{n(k)+1}\right)=b_{k} R \theta^{m-m}\left(b_{k}\right)=b_{k} R b_{k}$.

Since $b_{0}=a_{n(0)+1} \in a R \theta^{m}(a) \subseteq P(R), b_{0}$ is strongly nilpotent; hence $b_{k}=0$ and $a_{n(k)+1}=0$ for some $k \geq 0$.

Case 2. When the sequence $\left(s_{n}\right)_{n=0}^{\infty}$ is not bounded.

By symmetry we may assume that $\left(s_{n}\right)_{n=0}^{\infty}$ is not bounded above. So there is a strictly increasing sequence $(n(k))_{k=0}^{\infty}$ of positive integers such that $s_{n(0)} \geq 1$ and $s_{n(k+1)} \geq 1+2 s_{n(k)}$ for all $k \geq 0$. Let $z_{0}=s_{n(0)}$ and $z_{k+1}=s_{n(k+1)}-s_{n(k)}$ for all $k \geq 0$. Then $z_{0} \geq 1$ and $z_{k+1}=s_{n(k+1)}-s_{n(k)} \geq 1+s_{n(k)}$, and so $1+z_{0}+z_{1}+\cdots+z_{k}=1+s_{n(k)} \leq z_{k+1}$. Also let $b_{0}=a, b_{k+1}=a_{n(k)+1}$ for all $k \geq 0$. Then $b_{1}=a_{n(0)+1} \in a R \theta^{s_{n(0)}}(a)=b_{0} R \theta^{z_{0}}(a)$ and

$$
b_{k+1}=a_{n(k)+1} \in a_{n(k-1)+1} R \theta^{\left(s_{n(k)}-s_{n(k-1)}\right)}\left(a_{n(k-1)+1}\right)=b_{k} R \theta^{z_{k}}\left(b_{k}\right)
$$

for all $k \geq 1$. Since $b_{0}=a \in P_{\theta}(R), b_{0}$ is strongly $\theta$-nilpotent; hence $b_{k}=0$ and $a_{n(k)+1}=0$ for some $k \geq 0$.

Therefore $a$ is strongly $\left(\theta, \theta^{-1}\right)$-nilpotent.

The following, that is obtained from Corollary 3.8 and Lemma 3.9(2), (3), may be compared with [3, Proposition 1.11].

Corollary 3.10. $P_{\left(\theta, \theta^{-1}\right)}(R)=P(R) \cap P_{\theta}(R) \cap P_{\theta^{-1}}(R)$ and $P_{\left(\theta, \theta^{-1}\right)}(R)$ consists of all strongly $\left(\theta, \theta^{-1}\right)$-nilpotent elements in $R$.

The following theorem is shown by Proposition 3.7 and Corollary 3.10 . 
Theorem 3.11. (1) $P_{\sigma^{-1}}(S)=\left(P(R) \cap P_{\theta}(R) \cap P_{\theta^{-1}}(R)\right)[x ; \theta]$.

$$
\begin{aligned}
P(T) & =\left(P(R) \cap P_{\theta}(R) \cap P_{\theta^{-1}}(R)\right)\left[x, x^{-1} ; \theta\right] \\
& =\left(P(R) \cap P_{\theta}(R) \cap P_{\theta^{-1}}(R)\right)[x ; \theta] X^{-1} .
\end{aligned}
$$

Moreover, $P(T)$ is a graded ideal of $T$ and for $f(x)=\sum_{i=m}^{n} a_{i} x^{i} \in T$, $f(x) \in P(T)$ if and only if each $a_{i}$ is strongly $\left(\theta, \theta^{-1}\right)$-nilpotent in $R$.

Under some available conditions on $R$ and $\theta$, the prime radical of $R\left[x, x^{-1} ; \theta\right]$ is more tractable as in the following.

Corollary 3.12. (1) If $R$ satisfies the $A C C$ on $\theta$-ideals and $\theta^{-1}$-ideals, then $P(T)=P(R)\left[x, x^{-1} ; \theta\right]$.

(2) If $\theta$ is of locally finite order, then $P(T)=P(R)\left[x, x^{-1} ; \theta\right]$.

(3) If $\theta$ is $P Q I$ on $R$, then $P(T)=P_{\theta^{-1}}(R)\left[x, x^{-1} ; \theta\right] \subseteq P(R)\left[x, x^{-1} ; \theta\right]$.

Now we give an example of a QI automorphism $\sigma$ of $S$ such that $P_{\sigma^{-1}}(S) \varsubsetneqq$ $P(S) \varsubsetneqq P_{\sigma}(S)$.

Example 3.13. Let $R$ be a ring and $\theta$ be an automorphism of $R$ such that $P(R)=0$ and $P_{\theta}(R) \neq 0$ as in Example 2.2. Then $\sigma$ is a QI automorphism of $S$. In this situation we have $P_{\sigma^{-1}}(S)=\left(P(R) \cap P_{\theta}(R) \cap P_{\theta^{-1}}(R)\right)[x ; \theta]=0, P(S)=$ $P(R) \cap P_{\theta}(R)+\sum_{i=1}^{\infty} P_{\theta}(R) x^{i}=P_{\theta}(R)[x ; \theta] x$ and $P_{\sigma}(S)=\sum_{i=0}^{\infty} P_{\theta}(R) x^{i}=$ $P_{\theta}(R)[x ; \theta]$. Thus $P_{\sigma^{-1}}(S) \varsubsetneqq P(S) \varsubsetneqq P_{\sigma}(S)$.

\section{References}

[1] S. S. Bedi and J. Ram, Jacobson radical of skew polynomial rings and skew group rings, Israel J. Math. 35 (1980), no. 4, 327-338.

[2] J. Lambek, Lectures on Rings and Modules, Chelsea Publishing Co., New York, 1976.

[3] T. Y. Lam, A. Leroy, and J. Matczuk, Primeness, semiprimeness and prime radical of Ore extensions, Comm. Algebra 25 (1997), no. 8, 2459-2506.

[4] K. R. Pearson and W. Stephenson, A skew polynomial ring over a Jacobson ring need not be a Jacobson ring, Comm. Algebra 5 (1977), no. 8, 783-794.

[5] K. R. Pearson, W. Stephenson, and J. F. Watters, Skew polynomials and Jacobson rings, Proc. London Math. Soc. (3) 42 (1981), no. 3, 559-576.

Jeoung Soo Cheon

Department of Mathematics

Pusan National University

PusAn 609-735, Korea

E-mail address: jeoungsoo@pusan.ac.kr

EUN JEONG KIM

Department of Mathematics

Pusan National University

PUSAN 609-735, KOREA

E-mail address: ejkim69@pusan.ac.kr 
Chang IK LeE

Department of Mathematics

Pusan National University

PusAn 609-735, Korea

E-mail address: cilee@pusan.ac.kr

Yun Ho SHIN

Department of Mathematics

Pusan National University

PusAn 609-735, Korea

E-mail address: yyyi2000@yahoo.co.kr 